\title{
HER-2 Overexpression in Gastric and Gastroaesophageal Cancer: A Different Disease among Gastric Adenocarcinoma Subtypes
}

\author{
C Caglevic ${ }^{1}$, J Anabalon², S Panay², B Tudela ${ }^{3}$, C Gallardo ${ }^{4}$, M Mahave ${ }^{4}$, V Hornig and J Gallard $^{6}$ \\ ${ }^{1}$ Department of Medical Oncology, Universidad del Desarrollo, Chile
}

${ }^{2}$ Department of Medical Oncolgy, Fundacion Arturo Lopez Perez, Chile

${ }^{3}$ Radiotherapy Resident, Universidad de Valparaiso, Chile

${ }^{4}$ Medical Oncologist, Fundación Arturo López Pérez, Chile

${ }^{5}$ Medical Oncologist, Hospital Base San Jose Osorno, Chile

${ }^{6}$ Medical Oncologist, Clinica Indisa, Chile

Submission: September 13, 2018; Published: October 09, 2018

*Corresponding author: Christian Caglevic, Medical Oncologist, Medical Oncology Service, Department of Medical Oncology, Clinica Alemana Santiago, Av. Vitacura 5951, Vitacura, Santiago, Chile, Email: ccaglevic@alemana.cl / oncodemia@yahoo.com

\begin{abstract}
Gastric cancer and gastroesophageal junction adenocarcinoma is a common malignancy worldwide, in particular in Japan and in the Pacific coast of Latin America. Unless early diagnosis is performed, this disease carries a bad prognosis with a high mortality rate regardless of the use of standard treatments. A subgroup of this patients, probably close to a 20 percent, carry HER 2 mutations that result in HER 2 overepression. Targeted treatment to block HER 2 in the first line of treatment in the metastatic setting has resulted in positive outcomes when adding trastuzumab to standard chemotherapy, nonetheless no other HER 2 targeted treatments have reported benefit in this malignancy. Clinical trials are currently ongoing to evaluate the benefit of HER 2 blockade as perioperative treatment for patients that overexpress HER 2.
\end{abstract}

Keywords: Gastric Cancer; Gastroaesophageal cancer; Gastric; Gastroaesophageal adenocarcinoma; HER 2 overexpressed; Trastuzumab

\section{Introduction}

Gastric adenocarcinoma is currently one of the most frequent malignancies worldwide, becoming the fifth place in incidence and third place in mortality among solid tumors [1]. The geographical distribution of this disease is variable, being most common in countries with worse water quality and deficient food storage [2].

In the Pacific coast of South America, mainly in countries like Chile and Peru, probably due to a high prevalence of pathogenic Helicobacter Pylori subtypes, gastric cancer is a serious public health problem that involves a higher mortality when compared with developed countries of North America and Europe [3]. In high income countries, gastric cancer incidence has decreased during the last decades due to an important impact in environment that overlaps the genetic predisposition for this malignancy $[4,5]$.

Despite its high frequency, gastric adenocarcinoma, unfortunately, is often diagnosed at late stages, when curative surgery cannot be performed due to the presence of unresectable tumors or to metastatic disease. First steps of this malignancy are often asymptomatic or give mild and non- specific symptoms such as abdominal discomfort, anemia, weight loss, anorexia and others, which can delay the correct and opportune diagnosis [6].
This issue has been well considered in countries such as Japan, where gastric cancer screening has become a powerful tool in order to improve early diagnosis providing real options of curative treatments and therefore diminishing mortality $[7,8]$.

Regarding to non-metastatic locally advanced disease, different approaches to improve overall survival have been done. Despite that improving results have been achieved, several patients treated with a curative attempt will have disease recurrence and will die for this malignancy, including patients that underwent perioperative chemotherapy, post-operative radio-chemotherapy or adjuvant chemotherapy [9-11].

For metastatic gastric cancer the expected median overall survival is less than 1 year [12,13]. Since 1990's decade clinical trials have shown overall survival benefit when palliative chemotherapy has been compared with best supportive care $[13,14]$. Combining different cytotoxic chemotherapy agents has shown improving results in overall survival and higher response rates when compared with single agent chemotherapy, however these results are modest and adding a third drug does not really improve results but increases toxicity [15-19]. 


\section{Advanced Research in Gastroenterology \& Hepatology}

By recent years, thanks to a better comprehension of the molecular heterogeneity and of the pathways that are involved in gastric carcinogenesis, research has focused in new approaches such as targeted therapy [20]. Human Epidermal Growth Factor Receptor 2 (HER 2), also known as Cerb-2 o ERBB2, is a protooncogene located in chromosome 17 q21 that codes for this transmembrane protein that enhances a tyrosine kinase activity, conducting cell growth and differentiation [21]. HER 2 is an independent ligand that can be activated by mutations or due to overexpression of the receptor [21]. First discovered in breast cancer tumors, HER 2 mutations were found to have a worse prognosis of the disease when compared with patients that not carried such mutations [22,23]. Slamon et al. [24] shown that trastuzumab, a humanized IgG1 monoclonal antibody, when added to adjuvant chemotherapy regimen (doxorubicin plus cyclophosphamide follow by docetaxel), in Her 2 overexpressed patients, had an enormous impact in overall survival when comparing the chemotherapy regimen without adding the monoclonal antibody (HR 0.63; p<0.001) and also the arm that received trastuzumab had benefit in disease free survival (HR 0.64; $\mathrm{p}<0,001$ ) when compared with the control arm. Positive results were also achieved in HER 2 overexpressed breast cancer patients that underwent neoadjuvant treatment [25], and in metastatic disease when adding trastuzumab to chemotherapy or when including a double anti HER 2 blockade using also pertuzumab, other antibody that targets HER 2 overexpressed tumors [26,27].

Since recently, HER 2 mutations have been identified in several other solid tumors including ovarian cancer, prostatic cancer, lung cancer, colorectal cancer, gastric and gastroesophageal cancer as well [28].

Among gastric and gastroesophageal junction cancer patients, HER 2 overexpression has been found in different proportions depending of reports, therefore this incidence fluctuates between 4 to $53 \%$, with a median of incidence of $17,9 \%$ [28-30]. In Chile, where gastric cancer is a highly prevalent malignancy, HER 2 overexpression has been reported in the $11.9 \%$ of the patients. These findings have been reported mainly in the intestinal variety of adenocarcinoma but not in diffuse subtype patients [31].

HER 2 blockade in the first line of treatment for metastatic and unresectable gastric and gastroesophageal adenocarcinoma with HER 2 overexpression:

Looking forward improving survival in metastatic gastric cancer patients that overexpress HER 2, clinical trials have been conducted.

ToGA Trial was a phase III clinical trial for the first line of treatment of advanced gastric and gastroesophageal junction adenocarcinomas (unresectable locally advanced, recurrent and metastatic), restricted to patients with HER 2 overexpression in their tumors, that compared chemotherapy combination (fluoropyrimidine and cisplatin doublet) with or without adding trastuzumab [32]. This was a multicenter, open-label, randomized trial. From a total of 3807 patients that consented for participating in this study, only 810 overexpressed HER 2 (22\%), and finally a total of 584 patients from Asia, America and Europe, with measurable and non-measurable disease were randomized and treated. HER 2 expression of the tumors was assessed using different criteria as used for breast carcinoma, based on a different staining pattern of immunohistochemistry, which was also validated with FISH by Hofmann, including patients with HER $2+++$ in IHC or based on HER2:CEP17 ratio of at least 2 or greater [33].

Its primary endpoint was overall survival, showing a statistically and clinically relevant difference, with a mean overall survival of 13.8 months (95\% CI: 12-16) in those patients who received chemotherapy plus trastuzumab combination and 11.1 months (95\% CI: 12-16) in those patients who received chemotherapy without the monoclonal antibody, with a hazard ratio of 0.74 (95\% CI; 0.6-0.91 p=0.046). Secondary endpoints such as progression free survival, time to progression, duration of response and overall response rate were also higher in the trastuzumab plus chemotherapy combination group when compared with the control arm. In a post-hoc analysis, the effect on overall survival was greater on patients with higher levels of HER-2 expression (IHC 3+, IHC 2+ and FISH positive), achieving a median overall survival of 16 months versus 11 months in those with lower HER-2 expression (HER 2 1+- HER 2 negatives but FISH +). There were no differences in adverse effects between the control arm and study treatment arm, except for grade 3 infusion reactions that were higher in the trastuzumab group.

After these findings, the predictive value of HER 2 level was explored by Gomez-Martin and cols., by studying 90 patients with advanced gastric adenocarcinoma in the first-line treatment including trastuzumab, with regards of the HER2/CEP17 ratio and HER2 gene copies number (GCN), calculating a HER2/CEP17 ratio of 4.7 and GCN of 9.4 as the optimal cutoff levels for defining sensitivity to trastuzumab [34].

From a pharmacological point of view, there were differences in the calculated plasmatic clearance of trastuzumab between patients with HER-2 positive breast and gastric cancer, being higher on the latter, suggesting that a higher dose of trastuzumab would be more effective. This question was explored on a phase 3btrial (HELOISE, Hoffmann-La Roche) in the first line of treatment for gastric and gastroesophageal metastatic patients with HER 2 overexpression, that compared standard chemotherapy with two different doses of trastuzumab (loading dose of $8 \mathrm{mg} / \mathrm{kg}$ for both groups followed by $6 \mathrm{mg} / \mathrm{kg}$ intravenously every 3 weeks for control arm or $10 \mathrm{mg} / \mathrm{kg}$ intravenously every 3 weeks for the study arm). This trial was terminated because of futility demonstrated on a preplanned interim analysis [35].

Assuming the positive results previously achieved with trastuzumab, other strategies to block the HER 2 pathway were studied. The use of lapatinib, a small-molecule tyrosine kinase dual inhibitor of EGFR and HER 2, was explored in the phase III TRIO013/LOGiC trial, that compared chemotherapy (capecitabine plus oxaliplatin) with or without lapatinib in untreated patients with advanced or metastatic gastric, esophageal or gastroesophageal adenocarcinoma with HER 2-amplification assessed by FISH or, if not available, by immunohistochemistry (IHC 3+). Unfortunately, this trial failed to show benefit in overall survival and in progres- 
sion free survival when adding lapatinib in the study population. Nevertheless, in a preplanned subgroup analysis, the trial showed increased overall survival in Asiatic and in 60 years old or younger patients. Toxicity was higher in the lapatinib arm, principally more diarrhea grade 3 and higher was reported [36].

Looking forward enhancing HER 2 blockade, currently there is an ongoing phase III trial, JACOB, that compares the same study arm treatment used in ToGA trial (5FU or capecitabine, cisplatin and trastuzumab) with the addition of pertuzumab as a first line treatment for metastatic gastric and gastroesophageal gastric cancer that overexpresses HER 2. The primay end point of this study is to evaluate overall survival. Results have not been published yet [37].

\section{Perioperative chemotherapy in HER 2 overexpressed gastric and gastroesophageal adenocarcinoma with lo- cally advanced disease}

Considering the positive results of the ToGA trial, the next question to address is to evaluate if the addition of HER-2 targeted

\begin{tabular}{|c|c|c|c|c|c|c|c|c|}
\hline Reference & Country & Sex & Age & Stage & Chemotherapy & Surgery & pCR & Follow up \\
\hline Toshima et al. [40] & Japan & M & 60 & T4bN1M0 IIIB & $\begin{array}{c}\text { Cape/CDDP/T; S1/ } \\
\text { CDDP/T }\end{array}$ & $?$ & $?$ & $?$ \\
\hline Nishino et al. [41] & Japan & M & 61 & T4aN3M0, IIIc & S1/Cisplatin/T & $\mathrm{D}+\mathrm{D} 2$ & Yes & 10 months \\
\hline Segami et al. [42] & Japan & M & 67 & T4aN1M1(lung) & $\mathrm{XP}+$ Trastuzumab & & No, ypT4aN0M0 & 5 months \\
\hline Endo et al. [43] & Japan & M & 66 & $\begin{array}{c}\text { TxN3M1(liver, lymph } \\
\text { nodes) }\end{array}$ & $\begin{array}{c}\mathrm{XP}+\mathrm{T} \text { pre, } \mathrm{XT} \text { post, } \\
\text { T1a }\end{array}$ & & $\begin{array}{l}\text { No ypN0, yp- } \\
\text { M1(LYM) }\end{array}$ & 76 days \\
\hline Kanaya et al. [44] & Japan & M & 78 & T4a (SE) N2M0 IIIB & $\mathrm{XP}+\mathrm{T}$ preoperative & $\mathrm{T}+$ Spleen+D2 & No, T1b N0M0, IA & \\
\hline Li et al. [45] & China & $?$ & $?$ & TxNxM1 (liver) & S1+Oxali+T & $?$ & $?$ & 3 months \\
\hline Dong et al. [46] & China & M & 63 & TxNxM1 (adrenal) & XELOX + T, 3 cycles & $\begin{array}{l}\text { D+Adrenal- } \\
\text { ectomy+Cho- } \\
\text { lecistecto- } \\
\text { my+D2 }\end{array}$ & $?$ & 18 months \\
\hline Hyuga et al. [47] & Japan & M & 63 & TxNxM1 & $\begin{array}{l}\text { S1+P+T Progres- } \\
\text { sion S1 Docetaxel, }\end{array}$ & Distal & NO, T1a N0 M0 Ia & $?$ \\
\hline Yasuyama et al. [48] & Japan & $\mathrm{H}$ & 62 & $\begin{array}{l}\text { cT4b[Pancreas] } \\
\text { N3H0P0M0, IIIC }\end{array}$ & $\mathrm{S} 1+\mathrm{P}+\mathrm{T}$ & Distal+D2 & NO & $?$ \\
\hline Yasuyama et al. [48] & Japan & $\mathrm{F}$ & 62 & $\begin{array}{l}\text { cT4b [Pancreas] } \\
\text { N2H0P0M0, IIIC }\end{array}$ & $\mathrm{S} 1+\mathrm{P}+\mathrm{T}$ & Distal+D2 & NO & $?$ \\
\hline
\end{tabular}

Second line treatment blocking HER 2 for metastatic and unresectable gastric and gastroesophageal adenocarcinoma patients with HER 2 overexpression

The role of HER2 blockade in the metastatic second-line treatment scenario has been explored using two different agents: lapatinib, an oral tyrosine-kinase inhibitor of HER-1 and HER-2 intracellular domains, and ado-trastuzumab-emtansine (TDM-1), a monoclonal antibody conjugated of trastuzumab and emtansine, a cytotoxic microtubule binding agent. Both have shown activity on HER2 positive breast cancer beyond the first line of treatment in metastatic disease [48-52].

The TyTAN trial was an open-label multicenter phase III trial, including Asiatic patients, who had progressed after prior chemotherapy. It compared weekly paclitaxel with paclitaxel plus therapy in the perioperative setting can improve the outcomes in resectable - HER2 overexpressed gastric adenocarcinoma or allowing that unresectable disease to become potentially resectable in case of response. To answer this issue currently there are two ongoing clinical trials. INNOVATION trial is a randomized phase II collaborative study between EORTC and the Korean Cancer Study Group, comparing perioperative chemotherapy with fluoropyrimidine, cisplatin and trastuzumab, with or without the use of pertuzumab. Its primary endpoint is to evaluate and compare pathological complete response among study arms [38]. The other study, TRIGGER trial, is a randomized phase II trial, that compared perioperative chemotherapy with S-1 and cisplatin, with or without trastuzumab. Its primary endpoint is overall survival. [39].

Despite that there is not data yet the supports the use of HER 2 blockade in the perioperative setting, case reports have been published, most of them from Asiatic patients and centers [40-47] (Table 1).

lapatinib. There was no difference on overall survival, progression free survival or time to progression, showing effect only on overall response rate, which is of little clinical significance. In a subgroup analysis, there was benefit on overall survival in the IHC 3 + subgroup (median overall survival $5.6 \mathrm{~m}$ vs $4.2 \mathrm{~m}$; HR, 0.59; 95\% CI, 0.37 to 0.93 ; P 0.0176 ) and in Chinese population, when compared with Japanese, in median overall survival (9.7 m v 7.6 months; P 0.0351) and median progression free survival $(7.2 \mathrm{~m} \mathrm{v}$ 4.7 months ;P 0.0077) [53].

The GATSBY trial was an open-label phase 2-3 international multicenter study, that included advanced HER-2 positive gastric cancer who had progressed after a first-line therapy. It compared TDM-1 with taxane monotherapy (paclitaxel or docetaxel). The primary end point was overall survival with no significant differences between arms [54]. 
Both TyTAN and GATSBY raise the question about the different role of HER-2 in the gastric adenocarcinoma biology, in contrast to breast cancer, where sequential and/or additive blockade of HER2 signaling have some efficacy.

\section{Conclusion}

Gastric and gastroesophageal adenocarcinoma is a frequent malignancy that has an intrinsic bad prognosis unless its diagnosis was done in very early stages. HER 2 overexpression in tumor cells represent a worse prognosis, nevertheless blocking HER 2 has been demonstrated to be useful in the metastatic setting.

As an example, here, we report the case of a 65 years old man that in 2014 consulted due to dysphagia and loss of weight. Upper endoscopy found a subcardial tumor with compromise of the gastroesophageal junction. Its biopsy reported a gastric adenocarcinoma moderately differentiated. Baseline PET CT demonstrated multiple widespread disease beyond the primary tumor, metastasis was shown in lymph nodes, adrenal, lungs, liver and in several bones. After palliative radiotherapy to bone lesions to diminish bone pain, cytotoxic chemotherapy with FOLFOX regimen was started by June 2014. During cycle 4 of this regimen HER 2 was informed by IHC as highly overexpressed, then after trastuzumab was added with a loading dose of $8 \mathrm{mg} / \mathrm{kg}$ followed by $4 \mathrm{mg} / \mathrm{kg}$ every two weeks. After cycle number 22 of FOLFOX due to periferic neuropathy regimen was changed to $5 \mathrm{FU} /$ Leucovorin plus trastuzumab every two weeks. After completing a total of 35 cycles of chemotherapy (including both FOLFOX and $5 \mathrm{FU} / \mathrm{LV}$ regimens) and after achieving complete response patient has undergone continue treatment with trastuzumab $6 \mathrm{mg} / \mathrm{kg}$ every 3 weeks maintaining complete response. By date 62 cycles of trastuzumab have been administered without toxicity and with evident benefit (radiological and quality of life) (Figure 1).

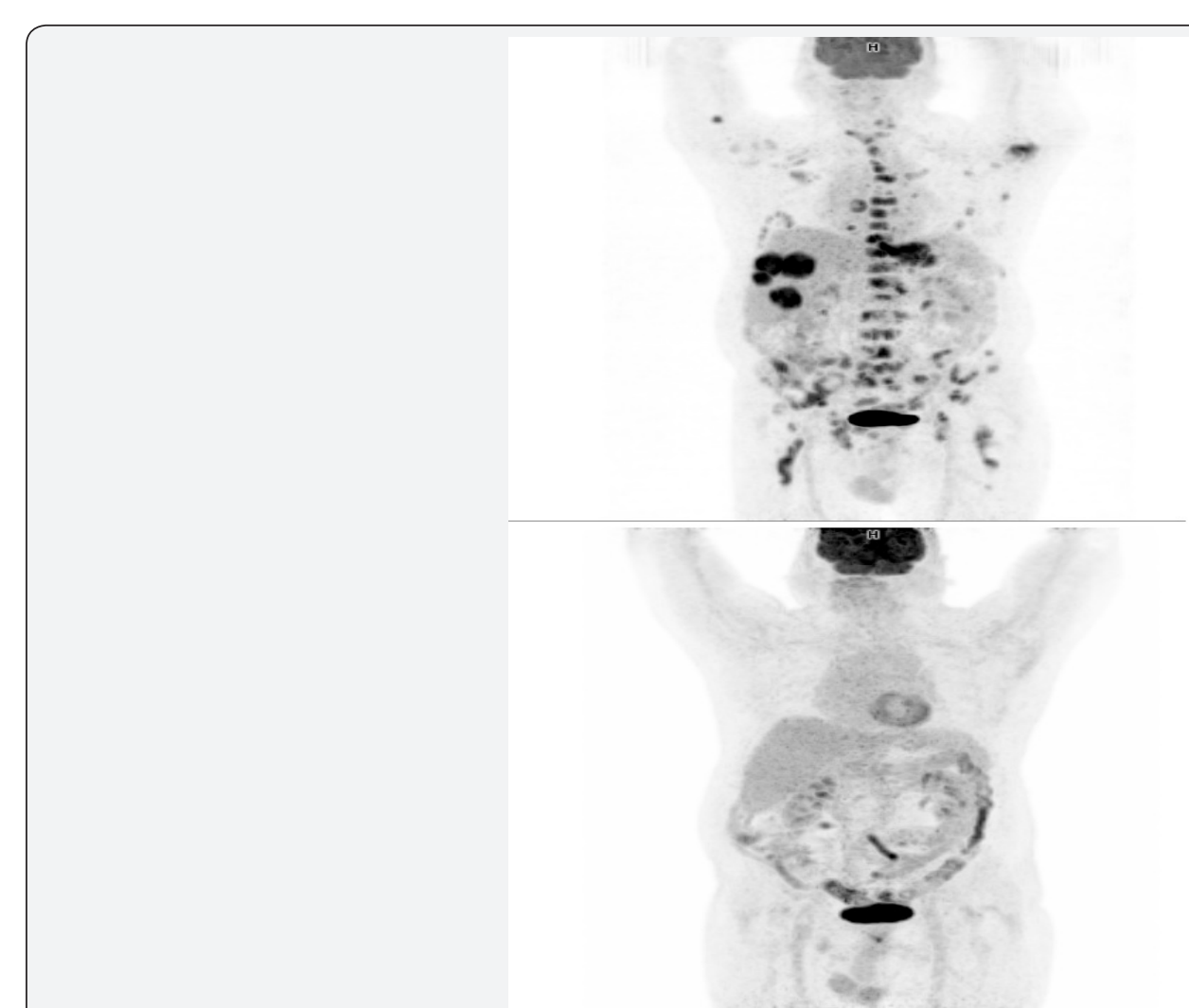

Figure 1: Above it is shown the PET CT image in the baseline situation of the patient with widespread disease due to metastatic HER 2 overexpressed metastatic gastric cancer. Below, the same patient after 3 years of treatment with trastuzumab and initially with combination with cytotoxic chemotherapy (FOLFOX followed by FU/LV), no evidence of metastatic disease has been found during the last year of maintenance treatment with single agent trastuzumab.

This case is an example because molecular profile, specifically HER 2, should be always be considered in the study of metastatic and or unresectable gastric cancer patients, and the use of trastuzumab in these cases should be mandatory unless contraindicated.

Despite the positive results of other HER 2 targeted treatment in the metastatic setting of breast cancer, no other targeted HER 2 blockades has shown real efficacy in gastric or gastroesophageal adenocarcinoma with HER 2 overexpression.
Clinical trials that are currently ongoing probably will answer if adding HER 2 blockades to perioperative and postoperative treatment in HER 2 overexpressed gastric and gastroesophageal junction adenocarcinomas will improve overall survival of these patients.

\section{Conflict of Interest}

Authors have not received funds neither any type of sponsorship and have not any conflict of interest regarding this manuscript. Dr C. Caglevic discloses: Speaker: BMS - MSD- Boehringer 
Ingelheim - Tecnofarma- ROCHE. Advisory and Consulting: BMS - MSD- Boehringer Ingelheim - Lilly - Astra Zeneca - Bayer. Investigator: MSD - BMS - Boehringer Ingelheim - Bayer- Roche- Astra Zeneca - Astellas- Advaxis. Travelling and educational grants: MSD - Boehringer Ingelheim, Dr C. Gallardo discloses: Travelling grants: MSD- Roche - Novartis - Eli Lilly. Advisory board: Eli Lilly - MSD.

\section{References}

1. Globocan 2012: Estimated cancer incidence, mortality and prevalence worldwide in 2012. IARC Publication.

2. Crew KD, Neugut AI (2004) Epidemiology of upper gastrointestinal malignancies. Semin Oncol 31(4): 450-464.

3. Caglevic C, Silva S, Mahave M, Rolfo C, Gallardo J (2016) The current situation for gastric cancer in Chile. Ecancermedicalscience 10: 707.

4. Parkin DM, Muir CS (1992) Cancer Incidence in Five Continents. Comparability and quality of data. IARC Sci Publ (120): 45-173.

5. Kubo A, Corley DA (2002) Marked regional variation in adenocarcinomas of the esophagus and the gastric cardia in the United States. Cancer 95(10): 2096-2102.

6. Correa P (2004) Is gastric cancer preventable? Gut 53(9): 1217-1219.

7. Khanderia E, Markar SR, Acharya A, Kim Y, Kim YW, et al. (2016) The Influence of Gastric Cancer Screening on the Stage at Diagnosis and Survival: A Meta-Analysis of Comparative Studies in the Far East. J Clin Gastroenterol 50(3): 190-197.

8. Hamashima C, Shibuya D, Yamazaki H, Inoue K, Fukao A, et al. (2008) The Japanese guidelines for gastric cancer screening. Jpn J Clin Oncol 38(4): 259-267

9. Cunningham D, Allum WH, Stenning SP, Thompson JN, Van de Velde CJ, et al. (2006) Perioperative chemotherapy versus surgery alone for resectable gastroesophageal cancer. N Engl J Med 355(1): 11-20.

10. Macdonald JS, Smalley SR, Benedetti J, Hundahl SA, Estes NC, et al (2001) Chemoradiotherapy after surgery compared with surgery alone for adenocarcinoma of the stomach or gastroesophageal junction. $\mathrm{N}$ Engl J Med 345(10): 725-730.

11. Noh SH, Park SR, Yang HK, Chung HC, Chung IJ, et al. (2014) Adjuvant capecitabine plus oxaliplatin for gastric cancer after D2 gastrectomy (CLASSIC): 5-year follow-up of an open-label, randomised phase 3 trial. Lancet Oncol 15(12): 1389-1396.

12. Cunningham SC, Kamangar F, Kim MP, Hammoud S, Haque R, et al. (2005) Survival after gastric adenocarcinoma resection: eighteen-year experience at a single institution. J Gastrointest Surg 9(5): 718-725.

13. Kamangar F, Dores GM, Anderson WF (2006) Patterns of cáncer incidence, mortality, and prevalence across five continents: defining priorities to reduce cancer disparities in different geographic regions of the world. J Clin Oncol 24(14): 2137-2150.

14. Murad AM, Santiago FF, Petroianu A, Rocha PR, Rodrigues MA, et al. (1993) Modified therapy with 5-fluorouracil, doxorubicin, and methotrexate in advanced gastric cancer. Cancer 72(1): 37-41.

15. Pyrhonen S, Kuitunen T, Nyandoto P, Kouri M (1995) Randomised comparison of fluorouracil, epidoxorubicin and methotrexate (FEMTX) plus supportive care with supportive care alone in patients with nonresectable gastric cancer. Brit J Cancer 71(3): 587-591.

16. Wagner AD, Grothe W, Haerting J, Kleber G, Grothey A, et al. (2006) Chemotherapy in advanced gastric cancer: a systematic review and meta-analysis based on aggregate data. J Clin Oncol 24(18): 2903-2990.

17. Cunningham D, Starling N, Rao S, Iveson T, Nicolson M, et al. (2008) Capecitabine and oxaliplatin for advanced esophagogastric cancer. New Engl J Med 358(1): 36-46.
18. Ross P, Nicolson M, Cunningham D, Valle J, Seymour M, et al. (2002) Prospective randomized trial comparing mitomycin, cisplatin, and protracted venous-infusion fluorouracil (PVI 5-FU) with epirubicin, cisplatin, and PVI 5-FU in advanced esophagogastric cancer. J Clin Oncol 20(8): 1996-2004.

19. Van Cutsem E, Moiseyenko VM, Tjulandin S, Majlis A, Constenla M, et al. (2006) Phase III study of docetaxel and cisplatin plus fluorouracil compared with cisplatin and fluorouracil as first-line therapy for advanced gastric cancer: a report of the V325 Study Group. J Clin Oncol 24(31): 4991-4997.

20. (2015) Targeted Therapies for Solid Tumors. In: A Russo, Rosell, Rafael, Rolfo, Christian, (Eds,) Current Clinical Pathology, Springer Science, Business Media, New York.

21. Akiyama T, Sudo C, Ogawara H, Toyoshima K, Yamamoto T (1986) The product of the human c-erbB-2 gene: a 185-kilodalton glycoprotein with tyrosine kinase activity. Science 232(4756): 1644-1646.

22. Yarden Y (2001) The EGFR family and its ligands in human cancer, signaling mechanisms and therapeutic opportunities. Eur J Cancer 37(Suppl 4): S3-S8.

23. Slamon DJ, Clark GM, Wong SG, Levin WJ, Ullrich A, et al. (1987) Human breast cancer: correlation of relapse and survival with amplification of the HER-2/neu oncogene. Science 235(4785): 177-182.

24. Slamon D, Eiermann W, Robert N, Pienkowski T, Martin M, et al. (2011) Adjuvant trastuzumab in HER2-positive breast cancer. N Engl J Med 365(14): 1273-1283.

25. Petrelli F, Borgonovo K, Cabiddu M, Ghilardi M, Barni S, et al. (2011) Neoadjuvant chemotherapy and concomitant trastuzumab in breast cancer: a pooled analysis of two randomized trials, Anticancer Drugs 22(2): 128-135.

26. von Minckwitz G, Schwedler K, Schmidt M, Barinoff J, Mundhenke C, et al. (2011) Trastuzumab beyond progression: overall survival analysis of the GBG 26/BIG 3-05 phase III study in HER2-positive breast cancer. Eur J Caner 47(15): 2273-2281.

27. Baselga J, Cortés J, Kim SB, Seock-Ah Im, Roberto Hegg, et al. (2012) Pertuzumab plus trastuzumab plus docetaxel for metastatic breast cancer. N Engl J Med 366: 109-119.

28. Yan M, Schwaederle M, Arguello D, Millis SZ, Gatalica Z, et al. (2015) HER2 expression status in diverse cancers: review of results from 37,992 patients. Cancer Metastasis Rev 34(1): 157-164.

29. He C, Bian XY, Ni XZ, Shen DP, Shen YY, et al. (2013) Correlation of human epidermal growth factor receptor 2 expression with clinicopathological characteristics and prognosis in gastric cancer. World J Gastroenterol 19(14): 2171-2178.

30. Jørgensen JT, Hersom M (2012) HER2 as a Prognostic Marker in Gastric Cancer - A Systematic Analysis of Data from the Literature. J Cancer 3: $137-144$.

31. Roa I, Jeannie Slater, Daniel Carvajal, Kurt Schalper, Gonzalo de Toro, et al. (2013) Expresión y amplificación del gen HER2 en el cáncer gástrico avanzado. Rev Med Chile 141(2): 1411-1419.

32. Bang YJ, Van Cutsem E, Feyereislova A, Chung HC, Shen L, et al. (2010) Trastuzumab in combination with chemotherapy versus chemotherapy alone for treatment of HER2-positive advanced gastric or gastro-oesophageal junction cancer (ToGA): a phase 3, open-label, randomised controlled trial. Lancet 376(9742): 687-697.

33. Hofmann M, Stoss O, Shi D, Büttner R, van de Vijver M, et al. (2008) Assessment of a HER2 scoring system for gastric cancer: results from a validation study. Histopathology 52(7): 797-805.

34. Gomez-Martin C, Plaza JC, Pazo-Cid R, Salud A, Pons F, et al. (2013) Level of HER2 gene amplification predicts response and overall survival in HER2-positive advanced gastric cancer treated with trastuzumab. J Clin Oncol 31(35): 4445-4452. 
35. (2000) A Study of Herceptin (Trastuzumab) in Combination with Cisplatin/Capecitabine Chemotherapy in Participants with Human Epidermal Growth Factor Receptor 2 (HER2)-Positive Metastatic Gastric or Gastro-Esophageal Junction Cancer (HELOISE). Clinical Trials.gov Bethesda (MD): National Library of Medicine (US).

36. Hecht JR, Bang YJ, Qin SK, Chung HC, Xu JM, et al. (2016) Lapatinib in Combination with Capecitabine Plus Oxaliplatin in Human Epidermal Growth Factor Receptor 2-Positive Advanced or Metastatic Gastric, Esophageal, or Gastroesophageal Adenocarcinoma: TRIO-013/LOGiC-A Randomized Phase III Trial. J Clin Oncol 34(5): 443-451.

37. (2000) A Study of Pertuzumab in Combination With Trastuzumab and Chemotherapy in Participants With Human Epidermal Growth Factor Receptor 2 (HER2)-Positive Metastatic Gastroesophageal Junction or Gastric Cancer. ClinicalTrials.gov. Bethesda (MD): National Library of Medicine (US).

38. (2016) Integration of Trastuzumab, with or without Pertuzumab, into Perioperative Chemotherapy of HER2- Positive Stomach Cancer: The INNOVATION Trial (EORTC-1203-GITCG). Oncol Res Treat 39(3): 153154.

39. Kataoka K, Tokunaga M, Mizusawa J, Machida N, Katayama H, et al. (2015) A randomized Phase II trial of systemic chemotherapy with and without trastuzumab followed by surgery in HER2-positive advanced gastric or esophagogastric junction adenocarcinoma with extensive lymph node metastasis: Japan Clinical Oncology Group study JCOG1301 (Trigger Study). Jpn J Clin Oncol 45(11): 1082-1086.

40. Toshima H, Hisamatsu A, Shimada K, Saito M, Suzuki M, et al. (2016) A Case of HER2-Positive Esophagogastric Junction Cancer with Perforation Curatively Resected after Neoadjuvant Chemotherapy plus Trastuzumab. Gan To Kagaku Ryoho 43(6): 761-764.

41. Nishino M, Hosoda Y, Okano M, Nagai K, Yasui M, et al. (2015) A Case of HER2-Positive Advanced Gastric Cancer with a Pathological Complete Response to Neoadjuvant Chemotherapy with S-1/CDDP/ Trastuzumab. Gan To Kagaku Ryoho 42(12): 2043-2045.

42. Segami K, Sato T, Kawabe T, Hayashi S, Makuuchi Y, et al. (2015) A Case of HER-2 Positive Advanced Gastric Cancer Responding to Capecitabine+Cisplatin+Trastuzumab Chemotherapy. Gan To Kagaku Ryoho 42(10): 1295-1297.

43. Endo S, Yamada T, Okuyama M, Hiraoka K, Konishi K, et al. (2015) A case of HER2-positive advanced gastric cancer successfully treated via capecitabine, cisplatin, and trastuzumab combination chemotherapy. Gan To Kagaku Ryoho 42(3): 359-361.

44. Kanaya N, Arata T, Tanakaya K, Yamasaki R, Aoki H, et al. (2014) A case of HER2-positive esophagogastric junction cancer treated by using a neoadjuvant chemotherapy regimen consisting of trastuzumab. Gan To Kagaku Ryoho. 41(12): 2285-2286.
45. Li ZY, Shan F, Zhang LH, Bu ZD, Wu AW, et al. (2014) Preoperative chemotherapy with a trastuzumab-containing regimen for a patient with gastric cancer and hepatic metastases. Genet Mol Res 13(4): 10952-10957.

46. Dong CX, Fu JF, Ye XY, Li XF, Zhong X, et al. (2014) Surgical resection of advanced gastric cancer following trastuzumab/oxaliplatin/ capecitabine combination therapy. World J Gastroenterol 20(34): $12355-12358$

47. Hyuga N, Kobayashi K, Watanabe R, Chono A, Matsumoto T, et al (2013) A case of SPT therapy(S-1+CDDP+trastuzumab) was successful for HER2-positive gastric cancer accompanied by distant lymph node metastasis. Gan To Kagaku Ryoho 40(12): 2256-2258.

48. Yasuyama A, Mikata S, Soma Y, Yukihiro Yoshikawa, Michiyo Kubota, et al. (2013) Two cases of human epidermal growth factor receptor 2-positive advanced gastric cancer successfully treated with $\mathrm{S}-1$, cisplatin, and trastuzumab combination therapy followed by curative resection. Gan To Kagaku Ryoho 40(12): 2203-2206.

49. Geyer CE, Forster J, Lindquist D, Chan S, Romieu CG, et al. (2006) Lapatinib plus capecitabine for HER2-positive advanced breast cancer N Engl J Med 355(26): 2733-2743.

50. Verma S, Miles D, Gianni L, Ian E Krop, Manfred Welslau, et al. (2012) Trastuzumab emtansine for HER2-positive advanced breast cancer. N Engl J Med 367: 1783-1791.

51. Krop IE, Kim S, González-Martín A, LoRusso PM, Ferrero JM, et al. (2014) Trastuzumab emtansine versus treatment of physician's choice for pretreated HER2-positive advanced breast cancer (TH3RESA): a randomised, open-label, phase 3 trial. Lancet Oncol 15(7): 689-699.

52. Wildiers H, Kim S, Gonzalez-Martin A, J-M Ferrero, R Yu, et al. (2016) Trastuzumab emtansine improves overall survival versus treatment of physician's choice in patients with previously treated HER2-positive metastatic breast cancer: Final overall survival results from the phase 3 TH3RESA study. Cancer Res 76(4).

53. Satoh T, Xu RH, Chung HC, Sun GP, Doi T, et al. (2014) Lapatinib plus paclitaxel versus paclitaxel alone in the second-line treatment of HER2-amplified advanced gastric cancer in Asian populations: TyTAN-a randomized, phase III study. J Clin Oncol 32(19): 2039-2049.

54. Thuss-Patience PC, Shah MA, Ohtsu A, Van Cutsem E, Ajani JA, et al. (2017) Trastuzumab emtansine versus taxane use for previously treated HER2-positive locally advanced or metastatic gastric or gastrooesophageal junction adenocarcinoma (GATSBY): an international randomised, open-label, adaptive, phase $2 / 3$ study. Lancet Oncol 18(5): 640-653.

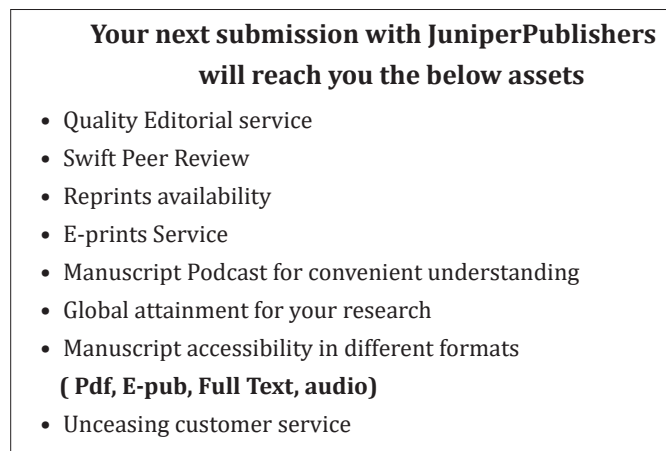

Track the below URL for one-step submission https://juniperpublishers.com/online-submission.php 